\title{
AN APPROXIMATE ANALYTICAL SOLUTION FOR THE DEPOSITION OF HEAVY PARTICLES RELEASED FROM AN ELEVATED LINE SOURCE
}

\author{
T. BOUVET* and J. D. WILSON \\ Department of Earth \& Atmospheric Sciences, University of Alberta, Edmonton, \\ Alberta, Canada, T6G $2 E 3$
}

(Received in final form 9 June 2005 / Published online: 28 December 2005)

\begin{abstract}
This work re-examines and further develops an analytical solution for the deposition swath of heavy particles released in the atmosphere from an elevated source over uniform terrain, correcting the particle diffusivity for the crossing trajectory effect. The revised (approximate) analytical solution proves to be accurate within $20 \%$ over a wide range of micrometeorological conditions and particle size, despite its neglect of the turbulence component of the deposition flux. It compares very satisfactorily with experimental data and with the simulations of a Lagrangian stochastic model, provided the variable $U(H) / w_{g} \leq 7$ (ratio of the mean horizontal wind speed at source height to the particle settling velocity). In this domain of validity, simple formulae relating the statistics of the deposition swath to $U(H) / w_{g}$ are derived.
\end{abstract}

Keywords: Analytical solution, Crossing trajectory effect, Diffusion, Heavy particle deposition, Lagrangian stochastic simulations, Turbulent deposition.

\section{Introduction}

In the context of particle dispersion in the atmosphere, numerical modelling is a powerful tool of investigation. However, the price of the flexibility it offers is a loss of physical transparency. An analytical solution offers two major advantages over numerical treatment: first, it gives direct insight to the parameters controlling dispersion and the related physics. Secondly, it is rapid and easy to use, whereas numerical simulation may be difficult. Thus, numerical and analytical treatments may be regarded as complementary.

Developing the work of Rounds (1955), Godson (1958) presented an analytical solution for the concentration field and the deposition swath of heavy particles released from an elevated line source over uniform terrain. The solution is founded on various assumptions, of which the strongest is to approximate

* E-mail: bouvet.thomas@gmail.com 
turbulent convection as a diffusion process, and with the heavy particle diffusivity equated with the eddy viscosity that is implicit in the logarithmic wind profile. Here we further develop the Rounds-Godson solution, modifying it to account for the crossing trajectory effect (Csanady, 1963). As we will show, simple adjustments to Godson's solution significantly improve its performance, and ensure satisfactory analytical results over a wide range of particle sizes and meteorological conditions. Our criteria for performance of the analytical solution include both experimental data (Hage, 1961; Walker, 1965) and numerical simulations using a Lagrangian stochastic (LS) model. The LS model is known (Wilson, 2000) to be fairly accurate for heavy particle dispersion over uniform terrain.

\section{The Analytical Solution}

\subsection{The Rounds-Godson SOLUTION}

In a steady-state regime, if one neglects streamwise turbulence and invokes gradient diffusion closure for the turbulent fluxes, then heavy particle transport and dispersion in a horizontally uniform flow can be described by the following mass conservation equation

$$
U \frac{\partial \chi}{\partial x}=\frac{\partial}{\partial z}\left(K \frac{\partial \chi}{\partial x}+w_{g} \chi\right)
$$

where $U=U(z)$ is the mean horizontal velocity, $\chi=\chi(x, z)$ is the particle concentration, $K=K(z)$ is the particle diffusivity and $w_{g}$ is the gravitational settling velocity of particles, namely the equilibrium velocity of a heavy particle falling in a static fluid. If the particle is spherical and small enough that Stokes' law holds, then $w_{g}=g \tau_{p}$, where $g$ is the gravitational acceleration and $\tau_{p}=\rho d^{2} / 18 \mu$ is the particle's acceleration time scale, $\rho$ being the density of the particle, $d$ its diameter, and $\mu$ the dynamic viscosity of the air. Here and henceforth, equations and variables are given (with a few obvious exceptions) in non-dimensional form, the source height $H$ and the friction velocity $u_{*}$ being taken as length and velocity scales.

Rounds (1955) derives an approximate solution of Equation (1) for an elevated line source releasing particles into a neutral (non-stratified) atmosphere. Godson (1958) generalizes Round's solution to the case of a thermally stratified atmosphere. The forms used for the profiles of vertical diffusivity $K(z)$ and horizontal wind speed $U(z)$ are derived from the (now obsolete, yet evidently useful) formulations suggested by Deacon (1949): 


$$
\begin{aligned}
U & =\frac{1}{k_{v}(1-v)}\left[\left(\frac{z}{z_{0}}\right)^{1-v}-1\right], \\
K & =k_{v} z_{0}^{1-v} z^{v},
\end{aligned}
$$

where $k_{v}$ is the von Karman constant and $z_{0}$ is the roughness length (defined as the height where the mean horizontal wind speed vanishes); $v$ is a stability parameter that relates to the Monin-Obukhov length $L_{\mathrm{mo}}$ as

$$
v=1-\frac{\ln \left(\varphi_{\mathrm{m}}\left(z / L_{\mathrm{mo}}\right)\right)}{\ln \left(z / z_{0}\right)}
$$

where $\varphi_{\mathrm{m}}$ is the Monin-Obukhov universal function for momentum; note that $v>1$ for unstable conditions, $v=1$ at neutral stability, and $v<1$ for stable conditions. The wind velocity profile (Equation (2)) satisfies $U\left(z_{0}\right)=0$ and tends to the logarithmic form $U=\left(1 / k_{v}\right) \ln \left(z / z_{0}\right)$ under neutral conditions, when $v \rightarrow 1$. No closed form solution to the heavy particle advection-diffusion Equation (1) can be achieved with the wind and diffusivity parameterizations (2)-(3), as such. However, the problem can be solved if the profiles (2)-(3) are approximated in the following manner:

$$
\begin{aligned}
U & =q\left(\frac{z}{z_{0}}\right)^{\alpha}, \\
K & =\epsilon k_{v} z,
\end{aligned}
$$

where

$$
\begin{aligned}
& \epsilon=\frac{2}{1+v} z_{0}^{1-v}, \\
& q=\frac{1}{k_{v} \alpha}\left(2 z_{0}\right)^{\alpha+v-1}, \\
& 1+\alpha=\gamma= \begin{cases}\frac{1-z_{0}^{1-v}}{\frac{1}{2-v}-z_{0}^{1-v}} & (v \neq 1) \\
\frac{\ln \left(z_{0}\right)}{1+\ln \left(z_{0}\right)} & (v=1)\end{cases}
\end{aligned}
$$

The parameters $\epsilon, \alpha$, and $q$ are determined by requiring that (in shorthand notation)

$$
\begin{aligned}
& \int_{0}^{1}(2) d z=\int_{0}^{1}(5) d z, \\
& \left.\frac{d(2)}{d z}\right|_{z=1 / 2}=\left.\frac{d(5)}{d z}\right|_{z=1 / 2}, \\
& \int_{0}^{1}(3) d z=\int_{0}^{1}(6) d z .
\end{aligned}
$$


Note that $z$ is dimensionless, so that $z=1$ is the source height. The bottom boundary of the domain ('the ground') is placed at $z=0$; it is assumed perfectly absorbing, and the diffusive flux to ground is neglected. Thus the lower boundary condition is specified by the deposition flux:

$$
D(x)=w_{g} \chi(x, z=0),
$$

or equivalently

$$
\left[K\left(\frac{\partial \chi}{\partial z}\right)\right]_{z=0}=0 .
$$

Based on the boundary condition (14)-(15) and on the profiles (5)-(6), a solution to Equation (1) is achieved. By application of Equation (14), the flux of particles to the ground normalized by the rate of emission $\mathrm{Q}$ is:

$$
\frac{D(x)}{Q}=\frac{1}{A \Gamma(p)} \exp \left(-\frac{A}{x}\right)\left(\frac{A}{x}\right)^{1+p},
$$

where

$$
\begin{aligned}
& p=\frac{w_{g}}{\epsilon k_{v} \gamma}, \\
& A=\frac{U(H)}{\epsilon k_{v} \gamma^{2}},
\end{aligned}
$$

and where $\Gamma(p)$ is the Gamma function of parameter $p: \Gamma(p)=\int_{0}^{\infty} t^{p-1} e^{-t} d t$. According to this solution, the deposition swath has the distribution of a Gamma function relative to the variable $A / x$, and with shape parameter $(p+2)$.

However, the solution of Godson actually omits an important feature of heavy particle dispersion, the 'Crossing Trajectory Effect' (henceforth called CTE) first identified by Csanady (1963). In the following paragraphs, after briefly recalling the physics underlying the CTE, a simple adjustment to the analytical solution, which allows us to account for the CTE, will be presented.

\subsection{AcCounting For the CROSSING trajectory EFFECt (CTE)}

By definition, velocity statistics of passive tracer particles are strictly identical to the velocity statistics of particles of the carrying fluid. However, heavy particles respond to gravity and to some extent fall out through the fluid, so as a result, the sample of fluid velocities 'seen' by heavy particles is not the same as the one seen by passive tracer particles. In other words, the velocity statistics of the driving fluid surrounding a particle differ, depending on whether this particle is passive or otherwise. In particular, the correlation time scale, which is a measure of the fluid velocity persistence along a particle trajectory, is altered. In the case of passive tracer particles, velocity de-correlation stems 


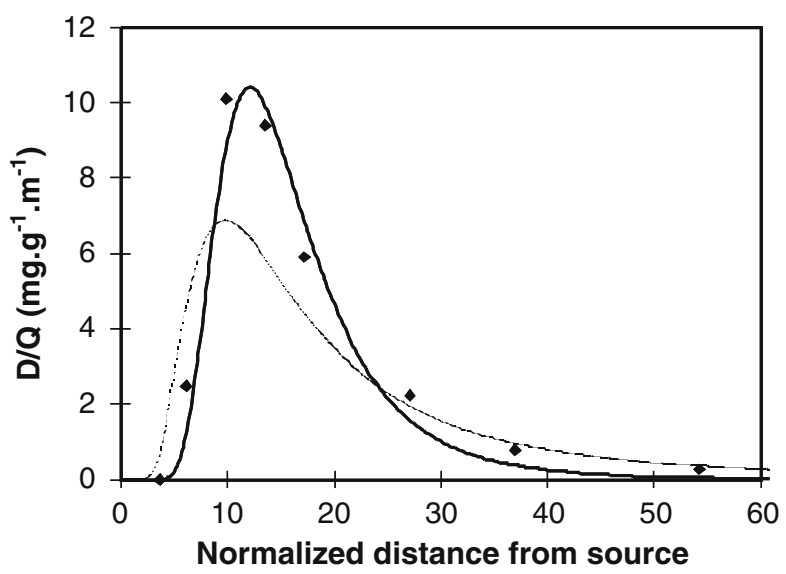

Figure 1. Normalized deposition rate of heavy particles (settling velocity $w_{g}=0.19 \mathrm{~m} \mathrm{~s}^{-1}$ ), released $7.4 \mathrm{~m}$ above ground, in a stable atmosphere $\left(u_{*}=0.18 \mathrm{~m} \mathrm{~s}^{-1}, z_{0}=0.016 \mathrm{~m}, L_{\mathrm{mo}}=16 \mathrm{~m}\right)$. Comparison between experimental data (dots) and analytical solutions in two cases: the solution of Godson, when the CTE is not included (dashed line), and the revised analytical solution, when the CTE correction is included (solid line).

only from eddy decay in time. Because heavy particles 'fall out' through eddies, the fluid velocity along their trajectories is additionally space-decorrelated. In consequence the velocity autocorrelation function for heavy particles $R_{\mathrm{HP}}(\epsilon)$, defined as $R_{\mathrm{HP}}^{i, j}=\overline{v_{i}(t) v_{j}(t+\epsilon)} /\left(\sigma_{v_{i}} \sigma_{v_{j}}\right)$, is expected to be smaller than its passive tracer particle counterpart $R_{\mathrm{L}}(\epsilon)$ when $\epsilon$ (the time of separation) is much larger than the particle inertial time scale $\tau_{p}$ (when $\epsilon \ll \tau_{p}$, then $R_{\mathrm{HP}}(\epsilon) \approx 1$ and $R_{\mathrm{HP}}(\epsilon) \geq R_{\mathrm{L}}(\epsilon)$; this is a consequence of inertia). The time scale for the dispersion of heavy particles (henceforth called $T_{\mathrm{HP}}=\int_{0}^{\infty} R_{\mathrm{HP}}(\epsilon) d \epsilon$ ) is accordingly reduced relative to the integral time scale $T_{\mathrm{L}}=\int_{0}^{\infty} R_{\mathrm{L}}(\epsilon) d \epsilon$. Figure 1 illustrates how critical the CTE can be in the dispersion of heavy particles; it presents experimental data for deposition, along with the corresponding analytical solution in two situations: when the CTE is accounted for (by the method described below), and when it is not (Godson's solution). Clearly, in this example, the CTE is significant, and accounting for it offers a major improvement in the analytical solution. We therefore propose below a simple adjustment to the eddy diffusivity, allowing CTE to be taken into consideration in the analytical solution.

To reconcile the eddy diffusion treatment with Taylor's (1921) Lagrangian treatment, one must require that the eddy diffusivity of tracer particles $K_{\mathrm{L}}$ satisfies ${ }^{1}$

$$
K_{\mathrm{L}}=\sigma_{w}^{2} \int_{0}^{\infty} R_{\mathrm{L}}(\epsilon) d \epsilon
$$

\footnotetext{
${ }^{1}$ In the interests of clarity, in the remainder of this section (only) we attach the subscript HP (or L) to the diffusivity for heavy particles (or tracer particles).
} 
In the far-field limit, when the travel time is much larger than $T_{\mathrm{L}}$, the particle diffusivity is linearly related to $T_{\mathrm{L}}$ :

$$
K_{\mathrm{L}, \infty}=\sigma_{w}^{2} T_{\mathrm{L}} .
$$

This result applies for the motion of passive tracer particles in the far-field of a source and in homogeneous turbulence. We will consider it as a pattern for the heuristic modification of heavy particle diffusivity $\left(K_{\mathrm{HP}}\right)$ in inhomogeneous turbulence, by writing

$$
K_{\mathrm{HP}, \infty}=\sigma_{w}^{2} T_{\mathrm{HP}} \text {. }
$$

Sawford and Guest (1991), following Csanady (1963), suggest that $T_{\mathrm{HP}}$ be parameterized in a heuristic fashion as

$$
T_{\mathrm{HP}}=\frac{T_{\mathrm{L}}}{\sqrt{1+\left(\beta \frac{w_{g}}{\sigma_{w}}\right)^{2}}},
$$

where $\sigma_{w}$ is the fluid vertical velocity variance and $\beta=\sigma_{w} T_{\mathrm{L}} / L_{\mathrm{E}}$ is an empirical parameter relating Eulerian and Lagrangian scales. By combining Equations (21) and (22), the heavy particle diffusivity in the far-field is

$$
K_{\mathrm{HP}, \infty}=K_{\mathrm{L}, \infty}\left[1+\left(\beta \frac{w_{g}}{\sigma_{w}}\right)^{2}\right]^{-1 / 2} .
$$

We justify this adjustment for $K_{\mathrm{HP}, \infty}$ by the evidence (see Figure 1) that it improves the agreement of the analytical solution with observations, and remind the reader that dispersion of particles in the near-field is outside the scope of this article (discussion on this point follows in Section 3.1). Therefore, we drop the far-field subscript $(\infty)$, on the understanding that the analytical solution is to be applied in the far-field of the source. As Godson showed, Equation (16) applies for any generalization of the eddy diffusivity, provided it can be written in the form (6); in particular, $\epsilon$ should not be a function of height. This requirement can be accommodated by writing

$$
\frac{K_{\mathrm{HP}}}{K_{\mathrm{L}}}(z) \approx \frac{K_{\mathrm{HP}}}{K_{\mathrm{L}}}(H)=\left[1+\left(\beta \frac{w_{g}}{\sigma_{w}(H)}\right)^{2}\right]^{-1 / 2} .
$$

Then the parameter $\epsilon$ becomes

$$
\epsilon=\varphi\left(\nu, z_{0}\right) \psi\left(\frac{\beta w_{g}}{\sigma_{w}(H)}\right),
$$

where $\varphi\left(v, z_{0}\right)$ is the adjustment made by Godson (Equation (7)) to generalize the diffusivity to stratified atmospheres, and $\psi\left(\frac{\beta w_{g}}{\sigma_{w}(H)}\right)$ is the additional correction for heavy particle diffusivity, presented in Equation (24). With this new 
formulation for $\epsilon$, the analytical solution (16) accounts approximately for both thermal stratification and for the crossing-trajectory effect.

\section{Discussion and Test of the Analytical Solution}

\subsection{ON THE ASSUMPTION OF 'FAR-FIELD' DISPERSION}

The formulation that is proposed for the eddy diffusivity $K$ (Equation (6)), and in turn the solution (16) as a whole, stands on the assumption that particles reach the 'far-field' of the source, i.e. that particle travel time is much larger than the velocity autocorrelation time scale $\left(T_{\mathrm{HP}}\right)$. Indeed, only by invoking a dependency of the eddy diffusivity on time (or distance) from the source can the advection-diffusion equation be forced to describe the near-field (eg. Deardorff, 1978). The 'far-field' assumption is however reasonable. Figure 2 shows the computed mean number $(N)$ of time intervals $T_{\mathrm{HP}}(t)$ that elapse during a particle's trajectory from the source to its point of deposition. Note that turbulence is not homogeneous in the boundary layer, and that $T_{\mathrm{HP}}$ decreases as the particle approaches the ground. Therefore, $T_{\mathrm{HP}}$ is not constant along a particle's trajectory. Were turbulence homogeneous (and therefore $T_{\mathrm{HP}}$ constant), $N$ would simply equal the ratio $t / T_{\mathrm{HP}}$ of the travel time of a particle to the velocity autocorrelation time scale. The mean $N$ may be loosely interpreted as the number of independent velocity 'choices' made by a particle along its flight, and one may consider the far-field to have been reached when $N \gg 1$. We see on Figure 2 that in standard meteorological conditions $\left(0.1 \leq u_{*} \leq 0.6 \mathrm{~m} \mathrm{~s}^{-1}\right), N \geq 7$ for particles $\left(w_{g} \leq 1.3 \mathrm{~m} \mathrm{~s}^{-1}\right)$ released from a source elevated at $H=0.1 \mathrm{~m}$. $N$ is larger when the source is higher (not shown), thus the assumption that particles reach the far-field is reasonable. It may lead to slight quantitative errors, in particular for large particles, but the analytical solution ought to capture at least the right qualitative behaviour.

\subsection{NeGLECT OF TURBULENT DEPOSITION}

Gravitational settling is the simplest and most obvious mechanism for deposition. However, the overall deposition rate may be affected by the fluid velocity fluctuations, and we will call the resulting contribution to deposition the 'turbulent deposition'. Brooke and Hanratty (1994) conceptualize the turbulent deposition in terms of three mechanisms: free-flight, turbophoresis, and turbulent diffusion. The total turbulent deposition flux is the sum of these three components, the importance of each one varying with the distance to ground. 


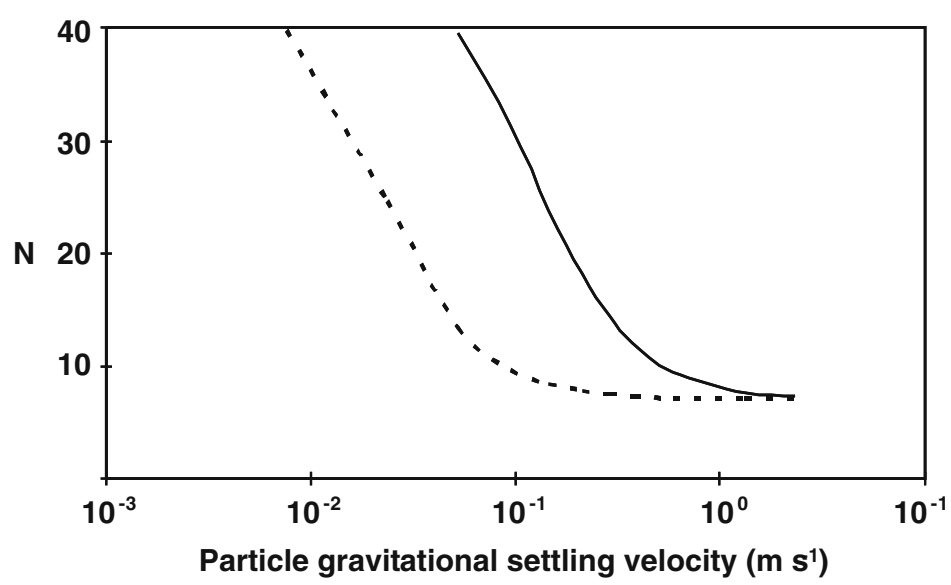

Figure 2. Estimation of the number $N$ of independent velocity choices made by a particle along its trajectory to its point of deposition, as a function of particle gravitational settling velocity $\left(w_{g}\right) . N$ has been computed with the LS model as the average number of elapsed intervals $T_{\mathrm{HP}}$ during the trajectory to deposition. The simulation is performed in a neutrally stratified atmosphere, with source height $H=0.1 \mathrm{~m}, z_{0}=0.01 \mathrm{~m}$ and $u_{*}=0.1 \mathrm{~m} \mathrm{~s}^{-1}$ (dashed line) or $u_{*}=0.6 \mathrm{~m} \mathrm{~s}^{-1}$ (solid line).

The solution of Rounds-Godson (16) is built on the assumption that gravitational settling is the only mechanism of deposition, as seen in Equations (14)-(15). To account for turbulent deposition, the lower boundary condition could appropriately be written

$$
D\left(x, z_{b}\right)=V_{\mathrm{dep}} \chi\left(x, z_{b}\right),
$$

where $z_{b}\left(\geq z_{0}\right)$ denotes the top of a shallow constant flux layer. As Slinn (1982) suggests, the deposition velocity $V_{\text {dep }}$ is the superimposition of the gravitational settling velocity $\left(w_{g}\right)$ and a component $\left(V_{\text {turb }}\right)$ ascribable to turbulent deposition:

$$
V_{\text {dep }}=w_{g}+V_{\text {turb }} .
$$

McCoy and Hanratty (1977) summarized the experimental results for turbulent deposition velocity collected by Friedlander and Johnstone (1957), Schwediman and Postma (1961), Wells and Chamberlin (1967), Farmer (1969), Schmel (1971), Forney and Spielman (1974) and Liu and Agarwal (1974). From this extensive data base, McCoy and Hanratty proposed the parameterization

$$
V_{\text {turb }}= \begin{cases}3.25 \times 10^{-4} \tau_{p}^{2} & 0.2<\tau_{p}<22.9 \\ 0.17, & \tau_{p} \geq 22.9\end{cases}
$$

where $V_{\text {turb }}$ and $\tau_{p}$ are non-dimensionalized with friction velocity and kinematic viscosity as scales. The case $\tau_{p} \leq 0.2$ is not relevant to this work, for the 


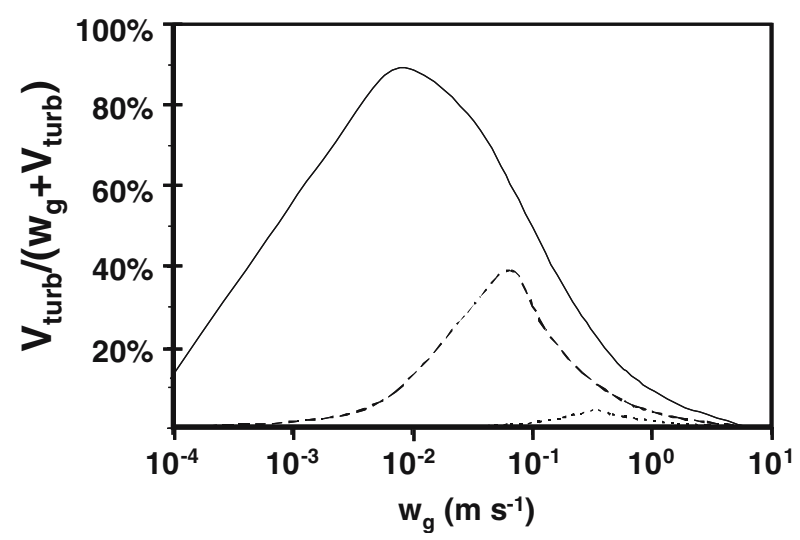

Figure 3. Fractional contribution of turbulent deposition $\left(V_{\text {turb }} \chi\right)$ to total deposition $\left(\left(V_{\text {turb }}+\right.\right.$ $\left.\left.w_{g}\right) \chi\right)$, as a function of particle gravitational settling velocity $\left(w_{g}=\tau_{p} g\right)$, when $u_{*}=0.6 \mathrm{~m} \mathrm{~s}^{-1}$ (solid line), $u_{*}=0.3 \mathrm{~m} \mathrm{~s}^{-1}$ (dashed line) and $u_{*}=0.1 \mathrm{~m} \mathrm{~s}^{-1}$ (dotted line). $V_{\text {turb }}$ is calculated according to Equation (28).

corresponding particles are submicronic and behave virtually like passive fluid particles.

Figure 3 presents the ratio $V_{\text {turb }} /\left(V_{\text {turb }}+w_{g}\right)$ as a function of $w_{g}$, where $w_{g}=g \tau_{p}$ and $V_{\text {turb }}$ is calculated according to Equation (28); evidently turbulent deposition can represent a significant component of total deposition. It was necessary therefore to assess the error induced by not accounting for turbulent deposition in the analytical solution of Rounds-Godson (i.e. Equation (16), which assumes Equations (14)-(15) at the lower boundary). This we did by comparing the analytical solution against finite difference numerical solutions of Equation (1) supplemented by the improved lower boundary condition (Equations (26)-(28)). Details are given in Appendix A. The results are shown in Figure 4, for a neutrally stratified atmosphere, and where $u_{*}=$ $0.35 \mathrm{~m} \mathrm{~s}^{-1}, H=1 \mathrm{~m}, z_{0}=0.01 \mathrm{~m}$. The small discrepancy between the numerical and the analytical solutions where they both assume the same (simpler) lower boundary condition (Equations (14)-(15)) shows that discretization errors in the numerical resolution are negligible. The numerical solution built with the lower boundary condition (26) is clearly distinguishable from the analytical solution; in particular, the peak deposition rate is noticeably stronger (i.e. ignoring turbulent deposition causes a reduced deposition peak). The most significant discrepancy between the Rounds-Godson solution and the numerical solution being the magnitude of the deposition peak, we present in Figure 5 the fractional error $E$ in this quantity (relative to the numerical solution taken as truth) as a function of $u_{*}, \tau_{p}$. It can be seen that $|E| \geq 20 \%$ when $u_{*} \geq 0.3 \mathrm{~m} \mathrm{~s}^{-1}$ and $w_{g} \leq 8 \times 10^{-2} \mathrm{~m} \mathrm{~s}^{-1}$. In this domain of the $w_{g}-u_{*}$ space, we consider that the analytical solution is unreliable. 


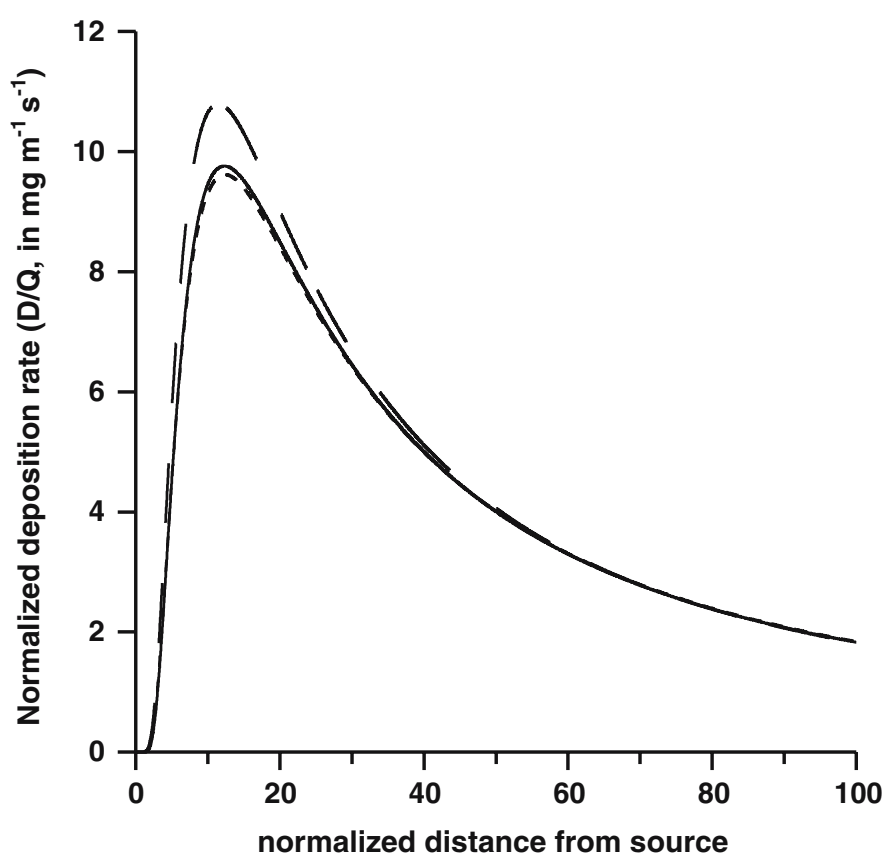

Figure 4. Deposition swath of heavy particles $\left(w_{g}=0.066 \mathrm{~m} \mathrm{~s}^{-1}\right)$ released at $H=1 \mathrm{~m}$ in a neutrally stratified atmosphere, where $u_{*}=0.35 \mathrm{~m} \mathrm{~s}^{-1}$ and $z_{0}=0.01 \mathrm{~m}$. The solid curve and the short-dashed curve represent respectively the analytical solution and the numerical solution of the advection-diffusion Equation (1) when turbulent deposition is ignored (lower boundary condition (14-15)). The long-dashed curve shows the numerical solution when turbulent deposition is included (lower boundary condition (26)).

\subsection{Test against experimental Results and LAgRangian stochastic SIMULATIONS}

Testing the analytical solution by comparing the deposition swath with its experimental or numerical counterpart can become confusing, especially when the number of comparisons is large. We therefore focus on four variables that characterize the deposition swath: the position $\left(x_{\text {peak }}\right)$ and magnitude $(D / Q)_{\text {peak }}$ of the deposition peak, the standard deviation $\left(\sigma_{x}\right)$ of the deposition location, and the distance from the source over which $90 \%$ of the particles will have been deposited $\left(x_{90 \%}\right)$. The analytical solution (16) implies that

$$
\begin{aligned}
x_{\text {peak }} & =\frac{A}{1+p}, \\
\left(\frac{D}{Q}\right)_{\text {peak }} & =\frac{[(1+p) / e]^{1+p}}{A \Gamma(p)}, \\
\sigma_{x} & =\frac{A}{p-1} \sqrt{\frac{1}{p-2}},
\end{aligned}
$$




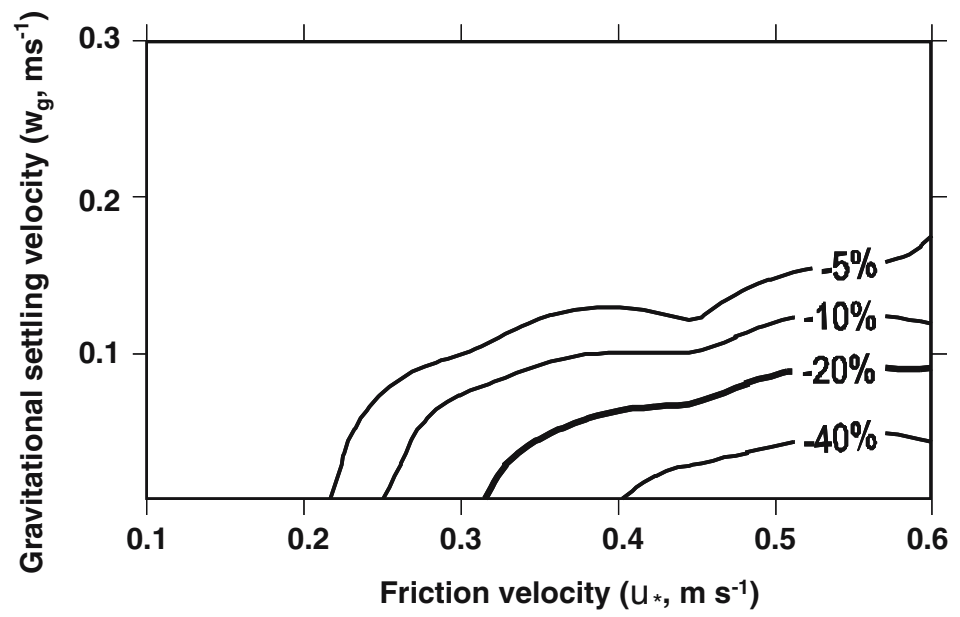

Figure 5. Fractional error in the approximate analytical solution, relative to a numerical solution of the advection-diffusion equation (Equation (1)) with the more realistic lower boundary condition (26-28). The other boundary conditions and the parameterizations for $\mathrm{U}$ and $\mathrm{K}$ are the same as assumed in the analytical treatment, and the atmosphere is taken as neutrally stratified. We found that the height of the source does not affect the distribution of the error in the $w_{g}-u_{*}$ space.

where $\sigma_{x}$ is defined analytically only for $p \in[2 ; \infty]$. Finally, the recovered fraction is related to the distance from the source by:

$$
R(x)=1-\frac{\Gamma_{A / x}(p)}{\Gamma(p)},
$$

where $\Gamma_{A / x}(p)$ is the incomplete Gamma function of parameter $p$, defined as

$$
\Gamma_{A / x}(p)=\int_{0}^{A / x} e^{-t} t^{-1+p} d t .
$$

The relationship $R(x)$ can be inverted numerically in order to obtain the recovery distance as a function of the corresponding recovered fraction.

We firstly test the refined analytical solution against the experimental data collected by Hage (1961) and Walker (1965) over a fairly broad range of micrometeorological conditions $\left(u_{*} \in[0.18,0.57] \mathrm{m} \mathrm{s}^{-1} ; H / L_{\mathrm{mo}} \in[-0.37,0.50]\right.$; $\left.z_{0} / H \in\left[5.4 \times 10^{-4}, 5.8 \times 10^{-3}\right] ; w_{g} / u_{*} \in[0.25 ; 1.66]\right)$. Figures 6 and $7 \mathrm{com}$ pare the analytical solutions for the position and the magnitude of the peak of the deposition swath, with their experimental counterparts. There is some uncertainty in the experimental values, for only 'samples' (discontinuous values) of the deposition swath are available. Thus, values for the peak location are interpolated; likewise, the values used for the magnitude of the peak are at best equal to the real values, and otherwise underestimations. In spite of the uncertainty inherent in the experimental data, one observes a very good match 


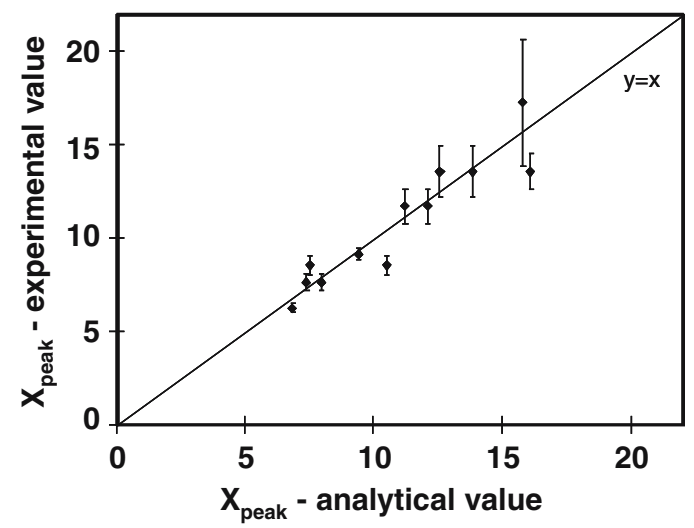

Figure 6. Comparison between the experimental value and the analytical solution for the position of the peak along the deposition swath. Conditions: $u_{*} \in[0.18,0.57] \mathrm{m} \mathrm{s}^{-1} ; H / L_{\mathrm{mo}} \in$ $[-0.37,0.50] ; \quad z_{0} / H \in\left[5.4 \times 10^{-4}, 5.8 \times 10^{-3}\right] ; \quad w_{g} / u_{*} \in[0.25,1.66]$.

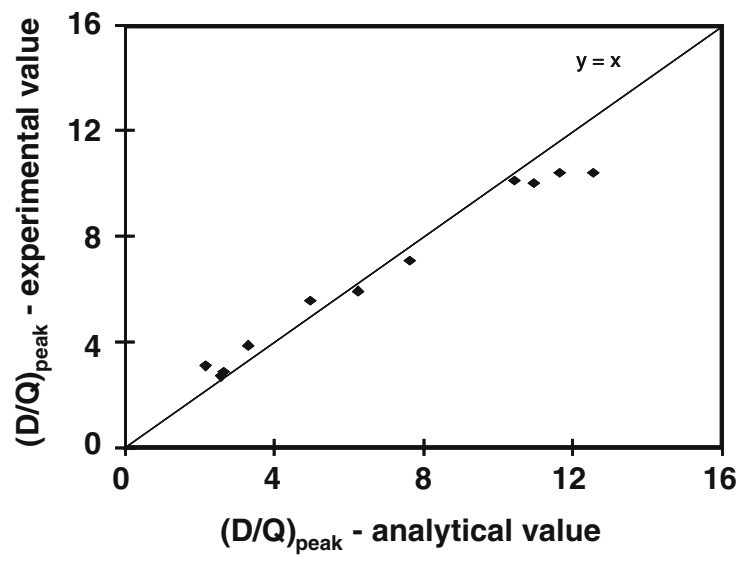

Figure 7. Comparison between the experimental value and the analytical solution for the normalized peak deposition rate. Conditions: $u_{*} \in[0.18,0.57] \mathrm{m} \mathrm{s}^{-1} ; H / L_{\text {mo }} \in$ $[-0.37,0.50] ; \quad z_{0} / H \in\left[5.4 \times 10^{-4}, 5.8 \times 10^{-3}\right] ; \quad w_{g} / u_{*} \in[0.25,1.66]$.

NB: Because experimental data are discontinuous along the deposition swath, the peak experimental value is likely to be an under-evaluation of the actual peak value.

between the theoretical and experimental values. This shows the satisfactory performance of the analytical solution, at least in the range of particle sizes used.

In order to test the analytical solution over a wider spectrum of conditions, we compare analytical results for the four swath statistics $\left(x_{\text {peak }},(D / Q)_{\text {peak }}, \sigma_{x}\right.$, $x_{90 \%}$ ) with corresponding predictions of a first-order LS model (for details of the model, please see Appendix B). These comparisons are performed over an extensive range of particle sizes and meteorological conditions $\left(u_{*} \in\right.$ $[0.1,0.5] \mathrm{m} \mathrm{s}^{-1}, H / L_{\mathrm{mo}} \in\{-0.4 ; 0.4\}, w_{g} / u_{*} \in[0.02,52.68]$, and the results are 
presented in Figures 8a-d. Each statistic $\left(x_{\text {peak }},(D / Q)_{\text {peak }}, \sigma_{x}\right.$ or $\left.x_{90 \%}\right)$ is plotted as a function of $U(H) / w_{g}$, resulting in a remarkably simple organization of the data, in both of the two (quite extreme) test situations of thermal stratification $\left(H / L_{\mathrm{mo}}=0.4\right.$ or $\left.H / L_{\mathrm{mo}}=-0.4\right)$. As far as $x_{\text {peak }}$ is concerned (Figure 8a), a discrepancy between the analytical and the LS model solutions becomes noticeable in the stable case for $U(H) / w_{g}>10$. For $(D / Q)_{\text {peak }}$ (Figure $8 \mathrm{~b}$ ), a growing discrepancy arises beyond $U(H) / w_{g} \approx 30$, in both cases of stratification. The graphic presenting $\sigma_{x}\left(U(H) / w_{g}\right)$ - Figure (8c) - is fairly incomplete, for $\left(\sigma_{x}\right)_{\text {analytical }}$ has a restricted domain of definition, as is clear by inspection of Equation (31), and furthermore $\left(\sigma_{x}\right)_{\mathrm{LS}}$ could not be computed with the LS model for large $U(H) / w_{g}$; in effect, under such conditions, a fraction of the particles released are deposited at a virtually infinite distance from the source. Since these particles are not deposited in the computational domain, the probability density function of the deposition location is incomplete, explaining why $\left(\sigma_{x}\right)_{\mathrm{LS}}$ could not be computed. However, in the restricted domain where $\sigma_{x}$ is defined and computed, the match between the analytical and LS model results is very satisfactory in both cases of stratification. Finally, Figure 8d shows how the analytical solution compares with LS results in terms of the recovery distance $x_{90 \%}$. In a stable atmosphere the match is almost perfect, even for very large values of $U(H) / w_{g}$. But in an unstable atmosphere, the analytical and LS model solutions diverge at $U(H) / w_{g}>7$. In summary, the match between the analytical solution and the more sophisticated LS model is excellent for the four criteria tested, as long as the variable $U(H) / w_{g}$ remains below 7. For larger values, the analytical solution gives a correct qualitative trend, but fails quantitatively in some conditions over one or more criteria.

\section{An Even Simpler Description Implicit in the Analytical Solution}

The value of $\beta$ is regarded as a constant in the following discussion, and set to unity, following Raupach (2002). In addition, since the analytical solution has been shown to be reliable only provided $U(H) / w_{g}<7$, the following discussion is restricted to this domain of validity.

According to the analytical formula for the deposition swath (Equation (16)), $A$ and $p$ are the driving variables. However, their interpretation is not simple. A dissection of parameters $A$ and $p$ allows us to retrieve the more fundamental variables $w_{g}, u_{\star}, v$ (the stability parameter) and $z_{0}$. The variable $U(H) / w_{g}$ in some sense integrates the influences of those 'fundamental parameters'. In addition, it allows a remarkable collapse of data for the four deposition swath characteristics. In other words, $U(H) / w_{g}$ properly accounts for most of the variability when all $z_{0}, v, u_{\star}$ and $w_{g}$ are variable. As an illustration, Figure 9 presents the relationship between $x_{\text {peak }}$ and $U(H) / w_{g}$ when the friction velocity, thermal stability, gravitational settling 

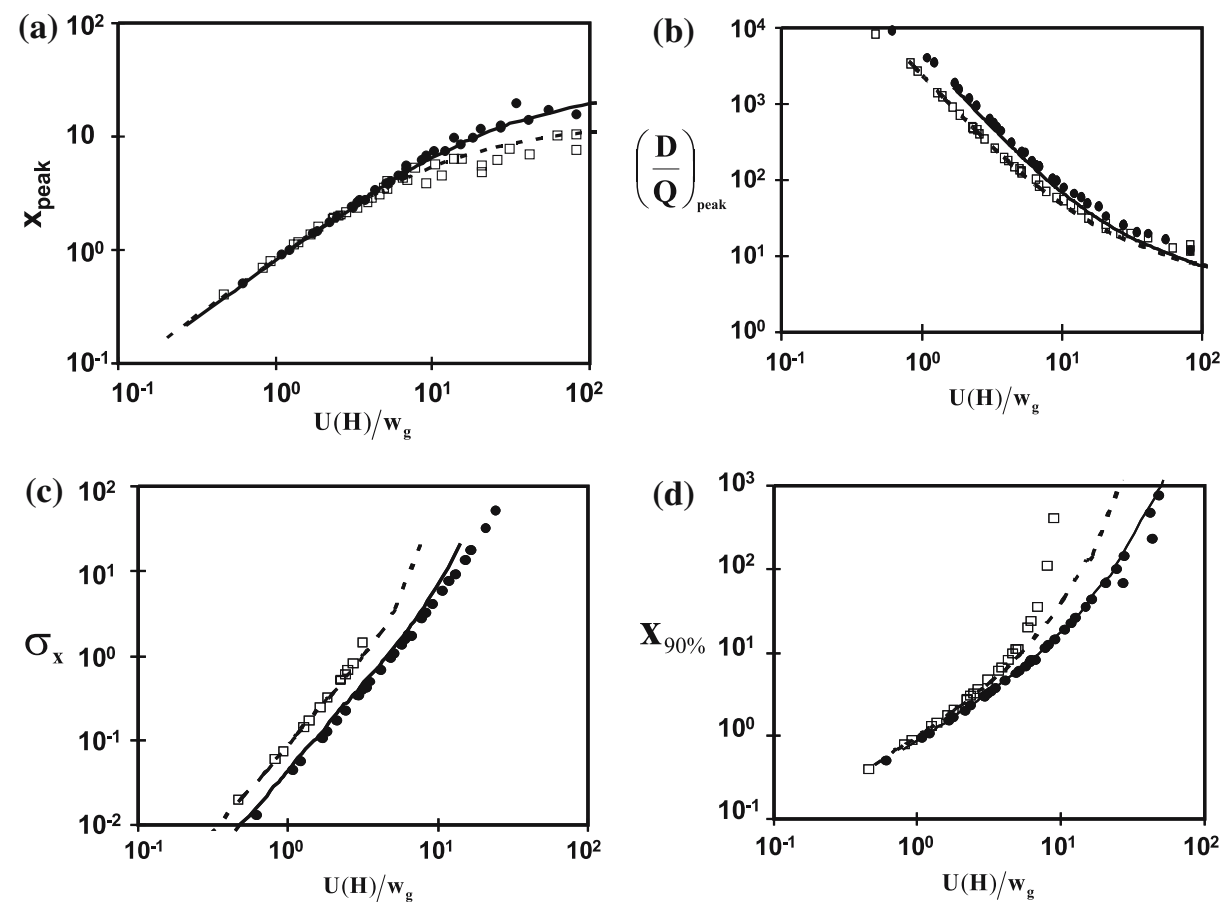

Figure 8. (a) Comparison between the analytical solution for the location of the deposition peak $\left(x_{\text {peak }}\right)$ and the corresponding LS solution. (b) Comparison between the analytical solution for the magnitude of the normalized deposition peak $\left([D / Q]_{\text {peak }}\right)$ and the corresponding LS solution. (c) Comparison between the analytical solution for the standard deviation of the deposition location $\left(\sigma_{x}\right)$ and the corresponding LS solution. (d) Comparison between the analytical solution for the ' $90 \%$ recovery distance' $\left(x_{90 \%}\right)$ and the corresponding LS solution. In Figures (a), (b), (c) and (d), the solid line and the dots present respectively the analytical and the LS model solutions in the stable case $\left(H / L_{\mathrm{mo}}=0.4\right)$. The dashed line and the squares present respectively the analytical and the LS model solutions in the unstable case $\left(H / L_{\mathrm{mo}}=\right.$ -0.4). Conditions: $u_{*} \in[0.1,0.5] \mathrm{m} \mathrm{s}^{-1} ; w_{g} / u_{*} \in[0.02,52.68] ; z_{0} / H=5 \times 10^{-3} ; \beta=1.0$.

velocity, and roughness length are all variable $\left(u_{\star} \in[0.1,0.5] \mathrm{ms}^{-1}, H / L_{\mathrm{mo}} \in\right.$ $\left.\{-0.4 ; 0.4\}, w_{g} / u_{\star} \in[0.02,52.68], z_{0} / H \in\left[2 \times 10^{-4}, 5 \times 10^{-2}\right]\right)$. The residual variability (i.e., the variability not 'explained' by $U(H) / w_{g}$ ) is seen in the width of the envelope that encompasses the cloud of dots. It is essentially due to the variability in $z_{0}$ when $U(H) / w_{g}<7$, and it remains small.

From a regression analysis, we derived the following relationships between the four statistics and $U(H) / w_{g}$ (in all cases, the fraction of variance explained, $R^{2}$, is larger than 0.990$)$ :

$$
x_{\text {peak }} \propto\left[U(H) / w_{g}\right]^{0.9},
$$




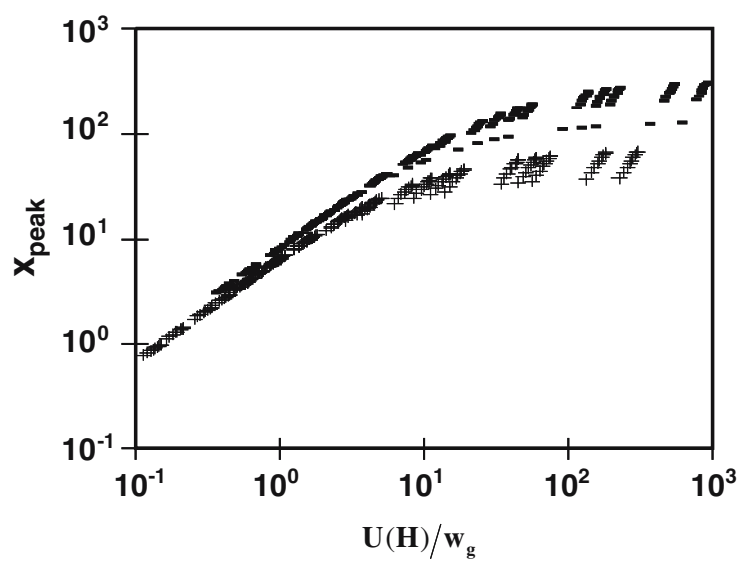

Figure 9. Analytical solution of the location of the deposition peak $\left(x_{\text {peak }}\right)$ over a wide range of conditions: $u_{*} \in[0.1,0.5] \mathrm{m} \mathrm{s}^{-1} ; w_{g} / u_{*} \in[0.02,52.68] ; H / L_{\mathrm{mo}} \in[-0.4,0.4] ; z_{0} / H=[2 \times$ $\left.10^{-4} ; 5 \times 10^{-2}\right] ; \quad \beta=1.0$. The 'dash' symbols (-) represent the situation $z_{0} / H=2 \times 10^{-4}$, whereas the 'plus' symbols (+) represent the situation $z_{0} / H=5 \times 10^{-2}$.

$$
\begin{aligned}
& \left(\frac{D}{Q}\right)_{\text {peak }} \propto\left[U(H) / w_{g}\right]^{-11 / 6}, \\
& x_{90 \%} \propto\left[U(H) / w_{g}\right]^{5 / 4}, \\
& \sigma_{x} \propto\left[U(H) / w_{g}\right]^{2} .
\end{aligned}
$$

Interestingly $x_{\text {peak }}$ is very well approximated by the ballistic impact point, defined as (here in dimensional form)

$$
x_{\text {bal }}=\int_{0}^{H / w_{g}} U\left(H-w_{g} t\right) d t
$$

i.e. $x_{\text {bal }}$ is the hypothetical location where a particle would deposit if it did not experience turbulence along its trajectory.

Equations (34)-(37) show that for growing $U(H) / w_{g}$, the characteristic length scales of the deposition swath (i.e. $x_{\text {peak }}, \sigma_{x}$ and $x_{90 \%}$ ) increase, whereas $(D / Q)_{\text {peak }}$ falls. Large $U(H) / w_{g}$ means that mean horizontal convection is stronger than mean vertical transport, thus a greatly extended deposition swath is no surprise. Large $U(H) / w_{g}$ also correlates with long time of flight before deposition, and therefore long action time for turbulence to disperse particles. This is consistent with the corresponding large spread (large $\sigma_{x}$ and small $\left.(D / Q)_{\text {peak }}\right)$.

\section{Conclusion}

A simple adjustment to the Rounds-Godson solution for heavy particle deposition to account for the CTE has significantly improved that solution, and could be easily 
transposed to the solution for the concentration field given by Rounds (1955). Tests and analysis of such a corrected solution will be addressed in a future work.

The refined analytical solution for deposition that we propose proves very skillful when $U(H) / w_{g}<7$. The distance of recovery, the location and intensity of the peak are all valuable information in the context of pollution management, and can all be calculated analytically. Consequently this solution may be of practical interest, for it is very easily accessible. It is also shown that the simple variable $U(H) / w_{g}$ exerts a key control on the deposition swath as a whole, and suffices to give a good estimate of it. However, the analytical solution is not suitable to handle dispersion over disturbed terrain, or from a complex source distribution; in such cases numerical modelling becomes necessary.

\section{Appendix A: Finite-Difference Solutions of the Advection-Diffusion Equation}

To test the consequence of neglecting turbulent deposition, we solved the advection-diffusion equation numerically with the contribution of turbulent deposition retained, using a regular, high-resolution mesh $(\triangle x=\triangle z=0.01 H)$ covering a computational domain sufficiently long $(100 H)$ to encompass most of the deposition swath and sufficiently high $(50 \mathrm{H})$ to ensure that the particle plume did not reach the top of the domain. An upwind difference was used for $\partial \chi / \partial x$, and a central difference was used for the vertical derivative (we followed the general approach of Patankar, 1980).

The source was placed at the entry boundary (index $i=0$ ) of the computational domain, where we prescribed a condition consistent with a line source, viz:

$$
\frac{\chi(0, j)}{Q}=\frac{1}{\triangle z U(j)} \delta_{j, j_{H}},
$$

where $j$ indexes gridpoint positions on the height axis and $j_{H}$ identifies the index coinciding most closely with source height. At the top of the domain

$$
\frac{\chi}{Q}\left(i, j_{\max }\right)=0 .
$$

No boundary condition was needed at the downwind $\left(i=i_{\max }\right)$ boundary, for due to the absence of diffusion on the $x$-axis this is a 'one-way' coordinate (Patankar, 1980). Parameterizations for $U(j)$ and $K(j)$ were identical to the ones used in the analytical treatment, i.e. Equations (5)-(6), with $\epsilon$ as in Equation (25). The numerical scheme was implicit on the $z$-axis, and the solution was obtained without iteration, simply by marching downwind on the $x$-axis away from the known inflow profile. When applied to the dispersion of passive tracer, this scheme essentially reproduces the observations of Project Prairie Grass (Barad, 1958). 


\section{Appendix B: The Lagrangian Stochastic Model}

To test the skills of the analytical solution, we performed simulations of particle dispersion using a first-order LS model. Such a model computes an ensemble of independent particle trajectories emanating from the source, by generating for each particle a time series of velocity and integrating it with respect to time. A statistically meaningful, smooth deposition swath is then inferred from a large ensemble of particle paths.

We used the LS model described by Wilson (2000) under the label 'Inertial Particle (IP) Model' (Section 2b of his paper). This model relies on two coupled equations: a Langevin equation gives the velocity of the 'driving fluid' that surrounds a heavy particle, and that velocity is imposed in the equation of motion for the particle's acceleration. The Langevin equation is adapted to surface-layer flow statistics and the Lagrangian time scale is corrected for the crossing trajectory effect following Sawford and Guest (1991); see Appendices (A, B) of Wilson (2000) for details. In order to best correspond with the analytical treatment being tested, the LS model was two-dimensional, and particles were always released at the same elevated location $H$. The boundary condition on the ground was also consistent across the two treatments, the ground being supposed to be a perfectly absorptive surface. Thus in the LS model a particle trajectory was terminated as soon as it reached the roughness height $z_{0}$, which means that the phenomenon of re-suspension was excluded.

\section{References}

Barad, M. L.: 1958, 'Project Prairie Grass, A Field Program in Diffusion', Geophysi. Res. Papers 59, I-III.

Brooke, J. W. and Hanratty, T. J.: 1994, 'Free-Flight Mixing and Deposition of Aerosols', Phys. Fluids 6(10), 3404-3415.

Csanady, G. T.: 1963, 'Turbulent Diffusion of Heavy Particles in the Atmosphere', J. Atmos. Sci. 20, 201-208.

Deacon, E. L.: 1949, 'Vertical Diffusion in the Lowest Layers of the Atmosphere', Quart. J. Roy. Meteorol. Soc. 75, 89-103.

Deardorff, J. W.: 1978, 'Closure of Second- and Third-Moment Rate Equations for Diffusion in Homogeneous Turbulence', Phys. Fluids 21, 525-530.

Farmer, R. A.: 1969, Liquid Droplets Trajectories in Two-Phase Flow, PhD thesis, Massachussets Institute of Technology.

Forney, L. J. and Spielman, L. A.: 1974, 'Deposition of Coarse Aerosols from Turbulent Flow', J. Aerosol Sci. 5, 257-271.

Friedlander, S. K. and Johnstone, H. F.: 1957, 'Deposition of Suspended Particles from Turbulent Gas Streams', Ind. Eng. Chem. 49, 1151-1156.

Godson, W. L.: 1958, 'The Diffusion of Particulate Matter from an Elevated Source', Arch. F. Met. Geophys. und Biokl. A 10, 305-327.

Hage, K. D.: 1961, 'On the Dispersion of Large Particles from a 15-m Source in the Atmosphere', J. Meteorol. 18, 534-539. 
Liu, Y. H. and Agarwal, J. K.: 1974, 'Experimental Observation of Aerosol Deposition in Turbulent Flow', J. Aerosol Sci. 5, 145-155.

McCoy, D. D. and Hanratty, T. J.: 1977, 'Rate of Deposition of Droplets in Annular Two-Phase Flow', Int. J. Multiphase Flow 3, 319-331.

Patankar, S. V.: 1980, Numerical Heat Transfer and Fluid Flow, Hemisphere Publ.

Raupach, M. R.: 2002, 'Diffusion of Heavy Particles in a Turbulent Flow', Geophys. Monograph., Environmental Mechanics: Water, Mass and Energy Tranfer in the Biosphere 129, American Geophysical Union, Washington, pp. 301-316.

Rounds, W.: 1955, 'Solutions of the Two-Dimensional Diffusion Equations', Trans. Amer. Geophys. Union 36, 395-405.

Sawford, B. L. and Guest, F. M.: 1991, 'Lagrangian Stochastic Simulations of the Turbulent Motion of Heavy Particles', Boundary-Layer Meteorol. 54, 147-166.

Slinn, W. G. N.: 1982, 'Predictions for Particle Deposition to Vegetative Canopies', Atmos. Environ. 16, 1785-1794.

Schmel, G. A.: 1971, 'Particle Diffusivities and Deposition Velocities over a Horizontal Smooth Surface', J. Colloid. Interface Sci. 37, 891-906.

Schwediman, L. C. and Postma, A. K.: 1961, 'Turbulent Deposition in Sampling Lines', USAEC Report No. HW-65309.

Taylor, G. I.: 1921, 'Diffusion by Continuous Movements', Proc. London Math. Soc. Ser. 2 20, 196-212.

Walker, E. R.: 1965, 'A Particulate Diffusion Experiment', J. Appl. Meteorol. 4, 614-621.

Wells, A. C. and Chamberlin, A. C.: 1967, 'Transport of Small Particles to Vertical Surfaces', Br. J. Appl. Phys. 18, 1793-1799.

Wilson, J. D.: 2000, 'Trajectory Models for Heavy Particles in Atmospheric Turbulence: Comparison with Observations', J. Appl. Meteorol. 39, 1894-1912. 